Journal of Thermal Engineering, Vol. 5, No. 3, pp. 149-156, April, 2019

Yildiz Technical University Press, Istanbul, Turkey

\title{
STUDY OF THE EFFECT OF THE POROUS PLATES ON THE TANK BOTTOM ON THE BOILING PROCESS
}

\author{
A. Nourbakhsh ${ }^{1}$, M. Bayareh",*
}

\begin{abstract}
In the present study, the boiling process is simulated in a fixed-temperature tank using the ANSYS FLUENT commercial software. Eulerian method is used to simulate the condensation and evaporation processes. The effect of porous media on the boiling process is investigated. For this purpose, a porous medium with different porosity coefficients is placed on the bottom of the tank and its effect on the bubble dynamics and wall thermal flux is investigated. The simulation is carried out in two dimensions and is considered as unstable. The results show that the porosity coefficient of the porous medium affects the bubble dynamics. The process of phase change decreases by decreasing porosity. Also, the growth rate of the bubble phase in the tank increases with increasing the heat transfer coefficient of the porous medium.
\end{abstract}

Keywords: CFD, Porosity Coefficient, Boiling, Two-Phase Flow

\section{INTRODUCTION}

Heat transfer has one of the most important engineering applications. The use of boiling heat transfer mechanism for cooling gives high heat transfer rate. If the temperature of the object immersed in the liquid, such as the wall of a container, is higher than the saturation temperature of the fluid, the pool boiling process occurs. The boiling process is characterized by the formation of bubbles, which are separated from the hot surface after growth. The porous medium is a solid material includes many holes. The fluid flows through the interconnected holes. The fluid path inside the porous medium is very complicated and therefore, the study on the microscopic scale is not possible. Porous media have a large contact surface with fluids, which can enhance the heat transfer effect. The porous medium not only changes the flow field conditions and causes the boundary layer to thinner, but also the conduction heat transfer coefficient becomes higher. Consequently, a porous medium placed in a fluid channel improves the thermal transfer properties efficiently. The reason for considering porous media is its application in a wide range of industrial such as geothermal systems, oil extraction, solid-state heat exchangers, thermal insulation, heaters, and journal bearings. So far, porous environments have been used extensively in industrial applications, including: Cooling of electronic components, geothermal systems, drying processes, heat exchangers. Many studies have been done to increase heat transfer using porous media. These studies include both experimental results and numerical simulations. Alkam et al. [1] studied forced heat transfer in fully developed region of canals with half volume fraction of porous material. They investigated the effects of different parameters such as the porosity layer thickness, the Darcy number, the thermal conductivity coefficient, and the microscopic inertial coefficient. The maximum Nusselt number is related to the duct filled with porous matter, and for inertial coefficients greater than 1000, the effects of the Darcy number are negligible. Kuznetsov [2] analyzed the previous problem for a fully developed flow and presented the critical parameters of heat transfer. He also studied the problem with the porous material at the center of the canal [3]. Jiang and Ren [4] studied the effects of viscous loss, boundary layer assumptions, heat dispersion, particle diameter, and variable oil properties on the heat transfer in a fully filled porous channel. It was concluded that heat transfer in a porous medium is numerically predictable. Angirasa [5] experimentally investigated the effect of high porosity (above 90\%) metal fibers in a wind tunnel through air flow. He showed that the use of metal porous material in comparison with a flat plate increases the heat transfer. Lee et al.

This paper was recommended for publication in revised form by Regional Editor Omid Mahian

${ }^{1}$ Department of Mechanical Engineering, Bu-Ali Sina University, Hamedan, Iran

${ }^{2}$ Department of Mechatronics Engineering, Shahrekord University, Shahrekord, Iran

*E-mail address: m.bayareh@sku.ac.ir

Orcid id: 0000-0002-7002-6092, 0000-0002-1821-3771

Manuscript Received 3 November 2017, Accepted 26 January 2018 
[6] studied the heat transfer in a channel filled with porous material and obtained the thickness of the boundary layer of the momentum as a function of the Reynolds and Darcy numbers. They provided two new equations for analyzing heat transfer for both solid and fluid phases. Mohamad [7] investigated the increase of heat transfer of laminar flow in a channel filled with all or part of it with porous material. He observed that by placing porous material in the center of the duct heat transfer increases and fully developed thermal length increases by $50 \%$ and even more. He also studied the effect of porous layer thickness on heat transfer and pressure drop. Kuznetsuv [8] numerically investigated the heat transfer of turbulent flow in a canal containing porous material that clings to the wall. He used the $k-\varepsilon$ turbulence model to calculate the roughness of the fluid interface with the porous material. He found that the Nusselt number has a minimum value relative to the position of the surface. Vafai and Haji-Sheikh [9] studied the heat transfer of a flow in a channel filled with porous medium. Channels had different cross-sections, in particular, they had parallel plates and also circular tubes were considered. Forooghi et al. [10] investigated the heat transfer in a stable and unstable pulse flow in a channel with porous material under the condition of thermal imbalance. In this research, the porous environment was in the wall and the walls were exposed to flux. They observed that increasing the conduction ratio increases the heat transfer. Satyamurti and Bhargavi [11] investigated the heat transfer in a thermal fully developed flow in a channel filled with porous material. It was concluded that the maximum heat transfer rate occurs in the porosity ratio of 0.8 at Darcy number equal to 0.001 . Similarly, the increase in heat transfer per pressure drop unit occurs in a porosity ratio of 0.7. Rudemiller et al. [12] studied a phase change in a porous medium. The results showed that the porous medium has a great influence on heat transfer enhancement. Liombus et al. [13] studied the dynamics of bubbles created by boiling water in porous media. This experimental study concluded that there is a relationship between gravity and heat transfer coefficient.

\section{GOVERNING EQUATIONS}

Flow and thermal fields are studied in a channel with a porous medium. Mass and heat transfer occur due to convection, molecular diffusion and turbulent eddies. The equations are applied to a control volume, which in these equations, $\mathrm{i}, \mathrm{j}, \mathrm{k}=1,2,3$ represents three dimensions. In the porous region, the effect of the presence of porous material is given in the momentum equation as loss terms. The physical properties of the porous material are presented as a function of porosity, fluid properties, and solid state properties. The governing equations are as follows:

Continuity equation:

$$
\frac{\partial}{\partial t}\left(\rho_{m}\right)+\nabla \cdot\left(\rho_{m} \vec{v}_{m}\right)=0
$$

where, $\vec{v}_{m}$ is weight averaged velocity and $\rho_{m}$ is density of mixture that are defined as follows, respectively:

$$
\begin{gathered}
v_{m}=\frac{\sum_{k=1}^{n} \alpha_{k} \rho_{k} \vec{v}_{k}}{\rho_{m}} \\
\rho_{m}=\sum_{k=1}^{n} \alpha_{k} \rho_{k}
\end{gathered}
$$

where, $\alpha_{k}$ is volume fraction of phase $k$.

Momentum equation:

$$
\begin{gathered}
\frac{\partial}{\partial t}\left(\rho_{m} \vec{v}_{m}\right)+\nabla \cdot\left(\rho_{m} \vec{v}_{m} \vec{v}_{m}\right)=-\nabla \cdot p+\nabla \cdot\left[\mu_{m}\left(\nabla \vec{v}_{m}+\nabla \vec{v}_{m}^{T}\right)\right]+ \\
\rho_{m} g+F+\nabla \cdot\left(\sum_{k=1}^{n} \alpha_{k} \rho_{k} \vec{v}_{d r, k} \vec{v}_{d r, k}\right)
\end{gathered}
$$


where, $\mu_{m}=\sum_{k=1}^{n} \alpha_{k} \mu_{k}$ and $\vec{v}_{d r, k}=\vec{v}_{k}-\vec{v}_{m} . p$ is the fluid pressure, $\rho$ is the fluid density, and $v$ is the kinematic viscosity. The porous medium also changes the pressure gradient by adding a source term to the right hand side of the Equation (4).

Energy equation:

$$
\frac{\partial}{\partial t} \sum_{k=1}^{n}\left(\alpha_{k} \rho_{k} E_{k}\right)+\nabla \cdot \sum_{k=1}^{n}\left(\alpha_{K} \vec{v}_{K}\left(\rho_{k} E_{k}+P\right)\right)=\nabla \cdot\left(k_{e f f} \nabla T\right)+S_{E}
$$

Porous media are modeled by adding a term to the standard fluid flow equations. This source term consists of two parts: a viscous loss (the first term in the right-hand side of equation (6) and an inertial loss term (the second term in the right-hand side of equation (6)).

$$
S_{i}=-\left(\sum_{j=1}^{3} D_{i j} \mu u_{j}+\sum_{j=1}^{3} C_{i j} \frac{1}{2} \rho|u| u_{j}\right)
$$

For a homogeneous environment:

$$
S_{i}=-\left(\frac{\mu}{K} u_{i}+C_{1} \frac{1}{2} \rho|u| u_{j}\right)
$$

$K$ is permeability and $C_{1}$ is the resistance of porosity medium. Indeed, the matrices $\mathrm{C}$ and $\mathrm{D}$ are two diagonal matrices with main diagonal of $C_{1}$ and $1 / K$, respectively.

For a laminar flow in a porous medium, the pressure drop is usually proportional to the velocity. So, the coefficient of $C_{1}$ can be ignored. By neglecting convection-diffusion acceleration, the porous media model is simplified by Darcy law [15]:

$$
\nabla P=-\frac{\mu}{K} \vec{u}
$$

So, the pressure drop in each directions $\mathrm{x}, \mathrm{y}$ and $\mathrm{z}$ inside the porous medium is as follows:

$$
\Delta P_{i}=\sum_{j=1}^{3} \frac{\mu}{K_{i j}} u_{j} \Delta n_{i} \quad, \quad i=x, y, z
$$

where, $\Delta n_{i}$ is the medium thickness.

Inside the porous medium, an effective thermal conductivity is used:

$$
\frac{\partial}{\partial t}\left(\varepsilon \rho_{f} E_{f}+(1-\varepsilon) \rho_{s} E_{s}\right)+\nabla \cdot\left(\vec{u}\left(\rho_{f} E_{f}+p\right)\right)=\nabla \cdot\left[k_{e f f} \nabla T-\left(\sum_{i} h_{i} j_{i}\right)+(\overline{\bar{\tau}} \cdot \vec{u})\right]+S_{f}^{h}
$$

In the above relation, $\varepsilon$ is the permeability of the medium, $\rho_{f}$ is the density of the fluid, $\rho_{s}$ is the density of the solid matrix of the porous medium, $E_{f}$ is the total energy of the fluid, $E_{s}$ is the total energy of the solid, $k_{e f f}$ of the effective thermal conductivity of the environment, temperature $\mathrm{T}, h_{i}$ and $j_{i}$ are enthalpy and flux of intrusion of $i$, respectively. $\overline{\bar{\tau}}$ is stress tensor, and $S_{f}^{h}$ is the source of the enthalpy of the fluid. $\overline{\bar{\tau}}$ is defined as:

$$
\overline{\bar{\tau}}=\mu\left[\left(\nabla \vec{u}+\vec{u}^{T}\right)-\frac{2}{3} \nabla \cdot \vec{u} I\right]
$$

The effective thermal conductivity of the porous medium is also calculated from equation (12): 


$$
k_{e f f}=(1-\varphi) k_{s}+\varphi k_{f}
$$

\section{RESULTS AND DISCUSSION}

The boiling process is simulated inside a tank (Fig. 1). The walls of the tank are insulated and part of the bottom has a constant temperature. The effect of porous media on the boiling process is investigated. In order to do that, the porous medium is placed with different porosity coefficients at different heights of the tank bottom. The effect of these parameters on the bubble dynamics is investigated.

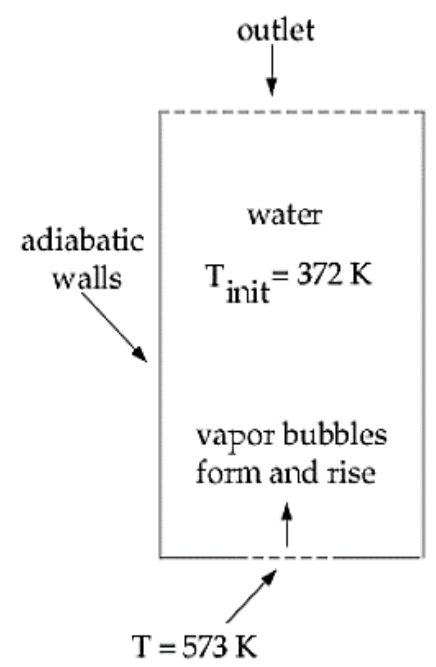

Figure 1. Schematic of the problem

Since the tank studied in this study is open, pressure outlet boundary condition is imposed on exit. All walls are assumed to be no-slip, so the velocity components on the walls are zero.

In order to investigate the effect of number of elements on the results, grid resolution tests are performed. The velocity profile is shown in Fig. 2 for different number of elements. According to this figure, with an increase in the number of elements to more than 27,000 cells, the velocity profile does not change significantly. Therefore, the grid with 27000 points is selected for further simulations.

In order to ensure the accuracy of the numerical solution method, the velocity distribution obtained from current simulation is compared with the results of reference [16] (Fig. 3). The figure shows that present simulations are in good agreement with the numerical results of [16].

After simulating the boiling process in a tank with a partial constant temperature of its lower surface, the effect of the porous medium (porosity coefficient) on the tank is investigated. The height of the porosity medium is $0.3 \mathrm{~m}$ and thermal conductivity is $4.24 \mathrm{~W} / \mathrm{m} . \mathrm{K}$. Cantor is the distribution of the volume fraction of steam, and the tank speed distribution.

The distribution of the volume fraction of the vapor phase is investigated at different conditions. Fig. 4 is presented to study the effect of porosity coefficient. It is found that by decreasing the porosity, the vapor phase fills more the reservoir and the rate of bubble generation increases. The reason is to facilitate the heat transfer process in the presence of porous medium by reducing the porosity coefficient inside the tank. So, the rate of phase change and the value of heat flux on the wall increases.

In order to investigate the effect of the porosity coefficient with the same thermal conductivity and identical height, Fig. 5 is presented. It is shown that the heat transfer increases with the decrease in the porosity coefficient results in an increases in the velocity of the vapor phase and in the boiling rate. 


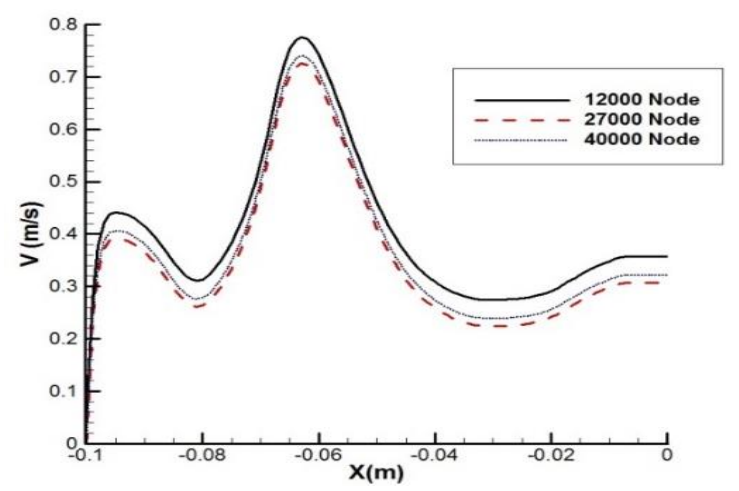

Figure 2. Grid study: velocity as a function of channel length

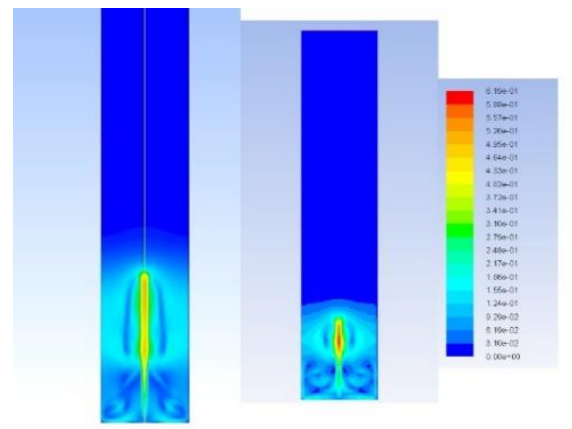

Figure 3. Comparison of velocity contours with the results of [16]. Left profile corresponds to present work

Now, the velocity profile at different cross-sections for different porosity coefficients is studied. The fluid velocity profile (mixture of vapor and saturated liquid) with three porosity coefficients of $0.5,0.7$ and 0.5 at different height from the bottom of the tank are considered (Fig. 6). The velocity of the fluid (mixture of vapor and saturated liquid) increases close to the bottom of the tank by decreasing the porosity. The boiling rate increases under these conditions. At high porosity coefficient, the fluid is approximately at rest and its velocity is zero. From the velocity distribution, it can be concluded that the vapor phase in the tank increases with decreasing the porosity. By comparing the volume fraction of the steam phase at different cross-sections of the tank (Fig. 7) for porosity coefficients of $0.5,0.7$ and 0.9 , it is confirmed that the reduction of the porosity coefficient causes an increase in boiling process. In a porous medium with a porosity coefficient of 0.9 , a volume fraction of the vapor phase is zero at the height of $0.5 \mathrm{~m}$ from the bottom of the tank, while at the same time, for a porosity coefficient of 0.5 , the vapor fraction of up to 0.2 is observed.

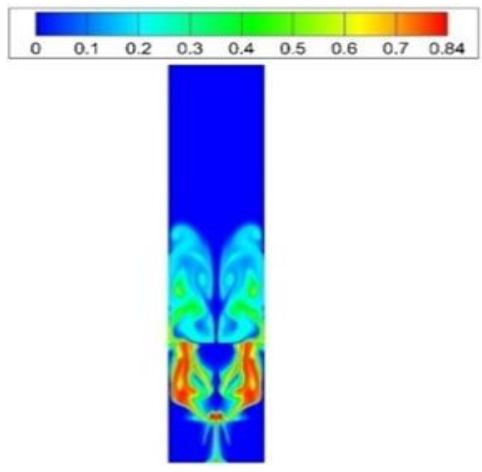

(a)

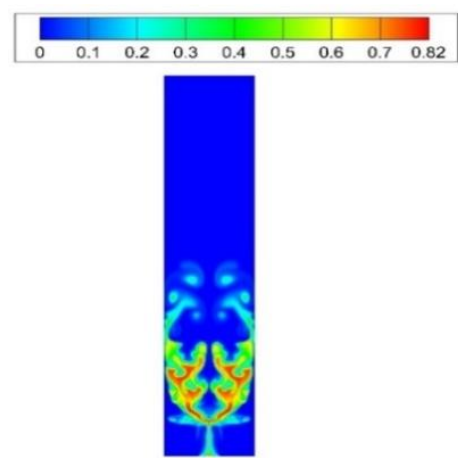

(b)

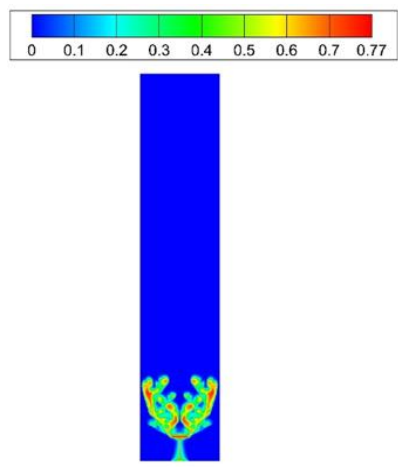

(c)

Figure 4. Distribution of vapor phase fraction in the environment with a porosity coefficient of (a) 0.5 (b) 0.7 , and (c) 0.9 
Journal of Thermal Engineering, Technical Note, Vol. 5, No. 3, pp. 149-156, April, 2019

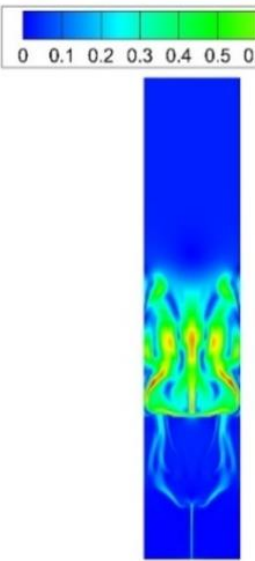

(a)
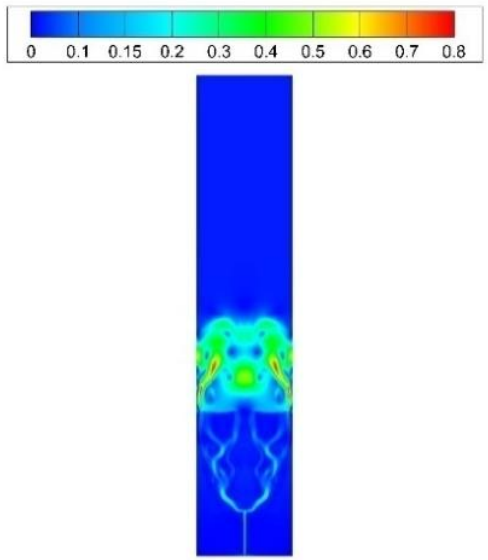

(b)

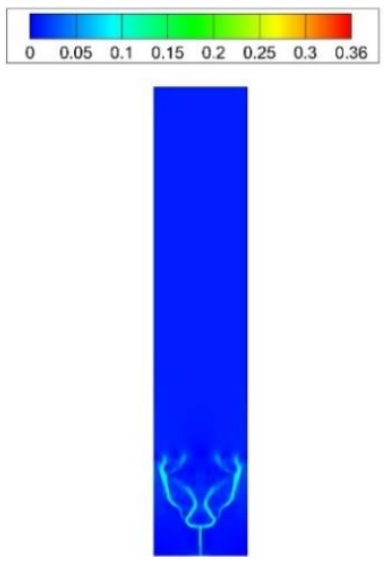

(c)

Figure 5. Velocity distribution of vapor phase in the environment with a porosity coefficient of (a) 0.5 (b) 0.7 , and (c) 0.9

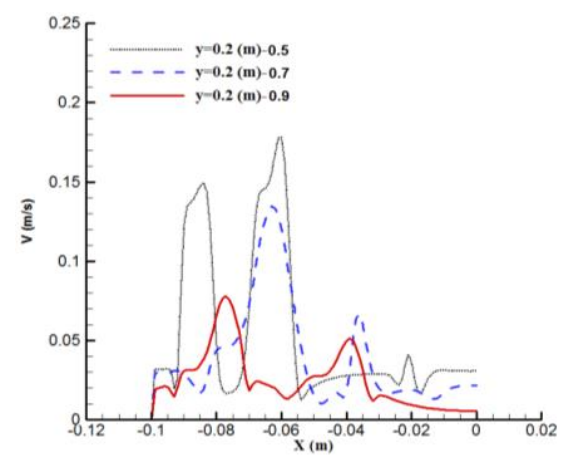

(a)

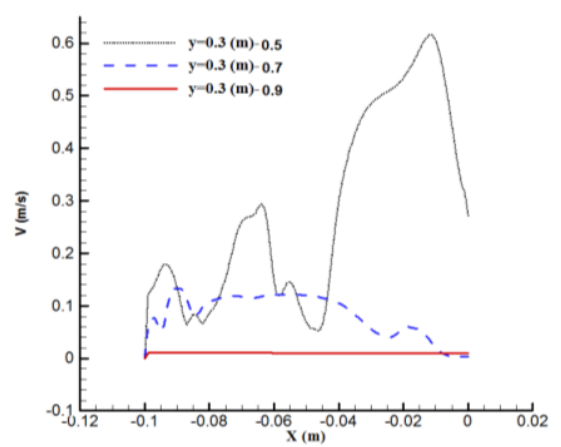

(b)

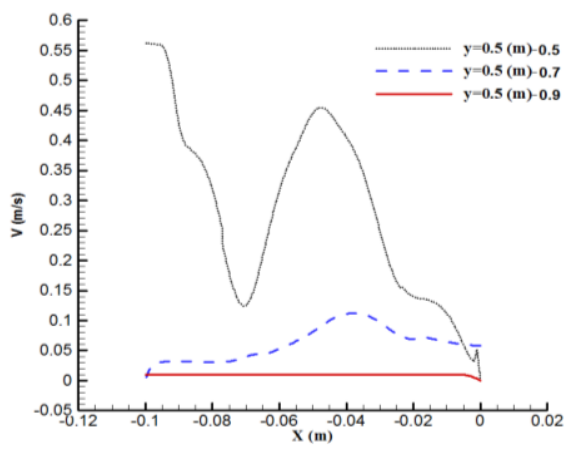

(c)

Figure 6. The velocity profile is in the range of (a) 0.1 , (b) 0.3 , and (c) 0.5 from the tank floor for three different porosity coefficients 


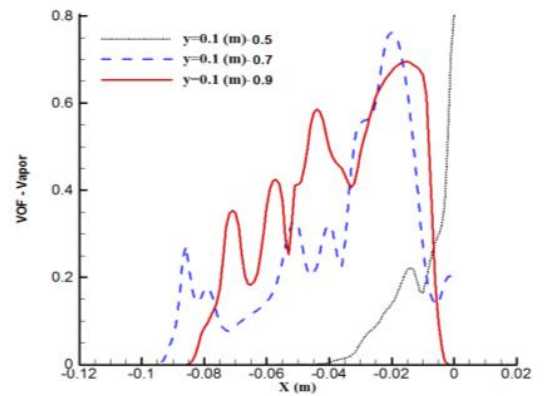

(a)

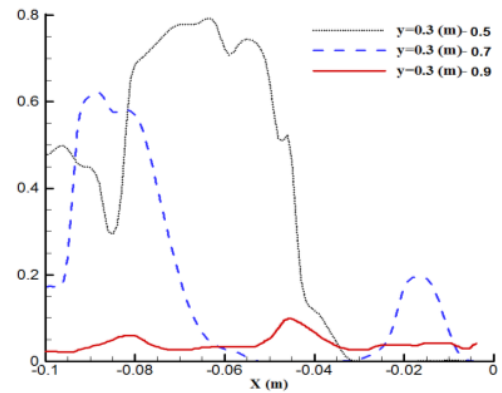

(b)

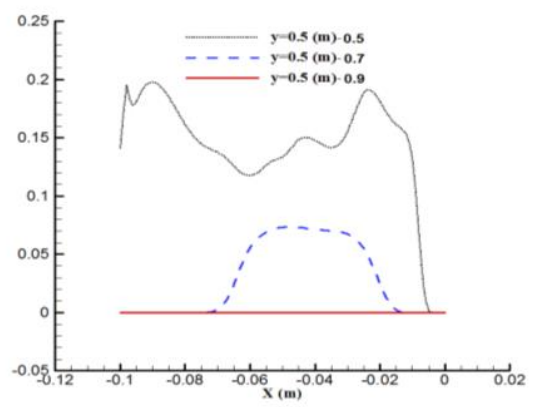

(c)

Figure. 7. The volume fraction of the vapor phase at a distance of (a) 0.1 , (b) 0.3 , and (c) 0.5 from the tank bottom for three different porosity coefficients

\section{CONCLUSIONS}

In the present study, using computational fluid dynamics, the boiling process of saturated water in a constant temperature tank with a porous medium is simulated and effect of porosity coefficient on bubble dynamics is investigated. The results showed that:

- The bubble generation rate in the tank increases with decreasing the porosity coefficient and decreasing convective heat transfer coefficient.

- The velocity of the fluid (mixture of vapor and saturated liquid) increases close to the bottom of the tank by decreasing the porosity.

- The reduction of the porosity coefficient causes an increase in boiling process.

\section{NOMENCLATURE}

E Energy

g Gravity Acceleration

h Enthalpy

P Pressure

T Temperature

$\mathrm{u} \quad$ Velocity component in the $\mathrm{x}$-direction

$\mathrm{v} \quad$ Velocity component in the y-direction

$\mathrm{x} \quad$ spatial coordinate component

y spatial coordinate component

$\alpha \quad$ Volume fraction

$\mu \quad$ Viscocity

$\rho \quad$ Density

$\tau \quad$ Stress tensor 
Journal of Thermal Engineering, Technical Note, Vol. 5, No. 3, pp. 149-156, April, 2019

\section{REFERENCES}

[1] M. K. Alkam, M. A. Al-Nimr, and M. O. Hamdan, (2001). Enhancing heat transfer in parallel plate channels by using porous inserts. International Journal of Heat and Mass Transfer, 44, 931-938.

[2] A. V. Kuznetsov, (1998). Analytical Study of Fluid Flow and Heat Transfer during Forced Convection in a Composite Channel Partly Filled with a Brinkman-Forchheimer Porous Medium. Flow, Turbulence and Combustion, $60,173-192$.

[3] A. V. Kuznetsov, (1999). Forced convection heat transfer in a parallel-plate channel with a porous core. International Journal of Applied Mechanical Engineering, 4, 271-290.

[4] P. X. Jiang, and Z. P. Ren, (2001). Numerical investigation of forced convection heat transfer in porous media using a thermal non-equilibrium model. International journal of Heat and Fluid Flow, 22, 102-110.

[5] D. Angirasa, (2002). Experimental investigation of forced convection heat transfer augmentation with metallic fibrous materialsll. International Journal of Heat and Mass Transfer, 45, 919-922.

[6] D. Y. Lee, J. S. Jin, J.S. and B. H. Kang, (2002). Momentum boundary layer and its influence on the convective heat transfer in porous media. International Journal of Heat and Mass Transfer, 45, 229-233.

[7] A. A. Mohamad, (2003). Heat transfer enhancements in heat exchangers fitted with porous media. Part I: constant wall temperature. International Journal of Thermal Science, 42, 385-395.

[8] A. V. Kuznetsov, (2004). Numerical modeling of turbulent flow in a composite porous/fluid duct utilizing a twolayer k-e model to account for interface roughness. International Journal of Thermal Science, 43, 1047-1056.

[9] K. Vafai, and A. Haji-Sheikh, (2004). Analysis of flow and heat transfer in porous media imbedded inside various-shaped ducts. International Journal of Heat and Mass Transfer, 47, 1889-1905.

[10] P. Forooghi, M. Abkar, and M. Saffar-Avval, (2011). Steady and unsteady heat transfer in a channel partially filled with porous media under thermal non-equilibrium condition. Transport in Porous Media, 86, 177-198.

[1] V. V. Satyamurty, and D. Bhargavi, (2010). Forced convection in thermally developing region of a channel partially filled with a porous material and optimal porous fraction. International Journal of Thermal Sciences, 49, 319-332.

[12] R. Rudemiller, J. D. Lindsay, (1989). Apparatus for investigating boiling phenomena in a fibrous porous medium. International Journal of Heat and Mass Transfer, 16, 785-794.

[13] J. S. Lioumbas, T. D. Karapantsios. (2015). Bubble dynamics and substrate thermalization during boiling in watersaturated porous matrix. Experimental Thermal and Fluid Science, 67, 75-80.

[14] K. Vafai, (2005). Handbook of Porous Media, 2nd edition, Taylor \& Francis Group, Boca Raton.

[15] Y. Ose, T. Kunugi, (2011). Numerical Study on Subcooled Pool Boiling. Nuclear Science and Technology, 2, $125-129$.

[16] Heat and Mass Transfer with the Mixture Model and Evaporation-Condensation Model, Tutorial Guide, Ansys 16. 